\title{
The geometry of confocal curves for passing through a door
}

\author{
Paolo Salaris, Christian Vassallo, Philippe Souères and Jean-Paul Laumond
}

\begin{abstract}
This paper presents a geometric approach to the problem of steering a robot subject to nonholonomic constraints through a door by using only visual measurements coming from a single fixed on-board monocular camera. The door is represented by two landmarks located on its vertical supports. After exploring the geometric structure that naturally emerges from the problem statement, e.g. bundle of hyperbolae, ellipses and circles, we exploit this planar geometry to provide stabilizing feedback control laws to drive the vehicle through the middle of the door. Using visual servoing we prove that this geometry can be directly measured in the camera image plane. Hence, we provide an image-based control scheme, avoiding the use of a state observer. Simulations in a realistic scenario and experiments are provided to show the effectiveness of the feedback control laws.
\end{abstract}

\section{INTRODUCTION}

This paper shows how a single camera is enough to design effective visual feedback control laws for a mobile robot to go through a door. The approach is derived from the natural geometry induced by the presence of a door in the environment, e.g. bundle of hyperbolae, ellipses and circles.

Visual servoing techniques are often used both to drive a robot towards a target, with and without obstacles and for indoor navigation, as e.g. along a corridor. For instance, in [1] two control strategies based on measurements coming from a pan camera and a 2D laser range sensor for steering the vehicle towards a target amidst obstacles has been provided. In [2] a landmark-based navigation approach among obstacles has been developed for humanoid robots. It integrates highlevel motion planning capabilities and a stack of feasible visual servoing tasks based on footprints following. The motion planning is based on the shortest path synthesis provided in [3], [4] where the limited Field-Of-View (FOV) problem is taken into account. In [5], [6] control laws to follow the shortest paths are also provided. In [7], a robust control strategy w.r.t. uncertainty on the depth of the target points and that takes into account the limits of actuator dynamics and the visibility constraint has been provided. On the other hand, in [8] authors provided a visual-based control strategy to steer the vehicle along a corridor by using the vanishing point defined by the intersection of the corridor guidelines. For the same problem, in [9] the optic flow was also used in combination with the perspective lines of the corridor. In [10] the temporal derivative of the optic flow has been exploited to determine the system state (orientation of the wall and distance to the wall), that is required to drive the robot through the center of the corridor.

This work was supported by European Research Council within the project Actanthrope (ERC-ADG 340050).

Authors are with LAAS-CNRS, 7 avenue du colonel Roche, F-31400 Toulouse Cedex 4, France.

e-mail: psalaris, cvassall, soueres, jplelaas.fr
Steering a vehicle through a door is one of the basic problem in visual servoing widely addressed in the literature. In [11] a framework for vision-based autonomous indoor navigation in a wheelchair capable of following a corridor and passing through doors using a single doorpost is provided. In particular, once a door is detected, the nearest doorpost is considered and a control laws able to steer the vehicle in front of the doorpost with a preassigned tolerance $m$ is provided. Then, a circular path of radius $m$ and centered at the doorpost is performed. In [12] door crossing is solved combining vision and ultrasonic sensor information. The robot approaches the door until an adequate distance is reached. Door traversing is then performed using sonar sensors. A similar problem has been solved in [13] for a large indoor surveillance robot equipped with a Kinect while crossing narrow doors. After detecting and locating the door, the robot is steered through it by a nonlinear adaptive controller. A sensor based algorithm for guiding a wheelchair through a doorway has been proposed also in [14]. The controller uses a camera and a laser range finder to perform the navigation. The problem of limited field-of-view constraints is also taken into account. In [15] the same problem is solved by dynamically generating Bézier-curve based trajectories while in [16] the door crossing problem in unknown environment for a wheelchair has been solved by a dynamic path planning algorithm based on successive points determination. Finally, in [17] authors propose a solution to a door crossing problem for an autonomous wheelchair equipped with a laser by solving a dynamic path planning algorithm based on successive points determination. An adaptive trajectory tracking control is then implemented to steer the wheelchair motion along the path in a smooth movement.

The approach used in this paper is different from the previous ones and the literature therein. Indeed, our method does not consist in a pre-planned path among via-points or a multi-stage strategy. On the contrary, we designed static feedback control laws (the vehicle velocities) that are functions of the current state of the system that is expressed in suitable coordinate systems that in turn can be directly measured in the image. As a consequence, the method avoids the computational cost of a state observer and the localization problem is automatically solved. Moreover, no delay is added due to the time of convergence of a state observer to the true values. The use of static feedback control laws instead of dynamic ones simplifies the implementation of our method and the analysis of the overall controlled systems in discrete time. Moreover, being a feedback and not a feedforward control or a planning, it intrinsically possesses robustness against disturbances and uncertainties. Finally, as our method does not need the depth information, only a fixed monocular camera is needed as opposed to several other approaches where the camera data are often fused with other sensor data as e.g. sonar and laser. 
In particular, the method we propose takes benefits from the geometry that naturally emerges from the problem statement. Seen from above, in the plane of the robot motion, the door is determined by two points: the footprints of its vertical supports. The originality of our approach is to introduce coordinate systems relative to these two points. The plane around the door is hence foliated by using confocal (the footprints of the door being the foci) hyperbolae and ellipses (a.k.a. elliptic coordinates system) and confocal circles that intersect at right angles (a.k.a. bipolar coordinates system). Using visual servoing we prove that these coordinates can be directly measured in the camera image plane. In other words, there exists a direct link between the geometry described by hyperbolae, ellipses and circles and the projection in the image plane of two landmarks located on the door supports and at the same height w.r.t. the the plane of the robot motion. We then provide feedback control laws based on these coordinate systems as well as proofs of asymptotic stability of the controlled system by using the Lasalle-Krasowskii principle. As both coordinates systems are immediately available in the image plane, we provide a so called Image-Based control schemes (see [18], [19] and [20]). As a consequence, neither a state observer nor other sensors, apart from the camera, are necessary to execute our visual servo control. Part of this work can be also found in [21]. In this paper we provide a more exhaustive description of control laws and strategies to drive the vehicle through a door, we extend the simulation part and we report in the appendix a complete analysis of the FOV constraints and strategies to cope with them. It is important to note that in this paper no door detector algorithm is considered, reasonably assuming that they will be always able to provide two points in the image plane corresponding to e.g. the top corners of the door. Moreover, the generalization of this method to other navigation problems between other type of landmarks is also possible. Both elliptic and bipolar coordinate systems are particularly appropriated to express the configuration of the vehicle w.r.t. any couple of points. In case of points at the same height from the plane of motion, they also provide the advantage of avoiding the use of a state observer - basically, in this case, elliptic and bipolar coordinates do not depend on the height of the landmarks but only on their positions in the image plane. For example, if it is possible, for any two obstacles, to identify two points at the same height, as for the door, the application of the proposed approach is straightforward also in obstacle avoidance problems. However, in general, it could be difficult to guarantee this assumption which is strictly related to the geometry of the environment around the vehicle. In these cases, the control laws developed in this paper can still be used as they assume that the heights of the landmarks are known constant parameters or they include a procedure to estimate them. Indeed, in case of landmarks at different heights, both bipolar and elliptic coordinates depend not only on the positions of the landmarks in the image plane but also on their heights.

The paper is structured as follows. The problem statement is given in section II. In section III elliptic coordinates are introduced and the direct link between these coordinates and the image plane is established. In section [V] the control law able to steer the vehicle through the door by using these coordinates is proposed, and the stability of the closed-loop system is shown. A second coordinates system, aka bipolar coordinates, is then introduced in section $\mathrm{V}$ and the link with the image plane is established. A control law to accomplish the task by using these coordinates is then provided in section $\mathrm{VI}$ and the stability of the controlled system is shown. In sections VII and VIII simulations and experiments to illustrate the effectiveness of our control laws are shown. Some conclusions and a resume of perspectives and open problems close the paper. Finally, in the Appendix the limited FOV problem is analyzed providing some strategies to overcome it.

\section{Problem StATEMENT}

Let us consider a vehicle moving on a plane where a righthanded reference frame $\langle W\rangle$ is defined with origin in $O_{W}$ and axes $X_{W}, Z_{W}$. The configuration of the vehicle is described by $q(t)=(x(t), z(t), \theta(t))$, where $(x(t), z(t))$ is the position in $\langle W\rangle$ of a reference point of the vehicle, and $\theta(t)$ is the vehicle heading with respect to the $X_{W}$ axis. Moreover, we assume that the dynamics of the vehicle is negligible. Using this notation, and denoting by $v(t)$ and $\omega(t)$ the robot linear and angular velocity, respectively, the kinematics exposes

$$
\left[\begin{array}{c}
\dot{x} \\
\dot{z} \\
\dot{\theta}
\end{array}\right]=\left[\begin{array}{cc}
\cos \theta & 0 \\
\sin \theta & 0 \\
0 & 1
\end{array}\right]\left[\begin{array}{c}
v \\
\omega
\end{array}\right] .
$$

This is the so-called unicycle in the literature [22]. The vehicle is equipped with a rigidly fixed pinhole camera with reference frame $\langle C\rangle=\left\{O_{c}, X_{c}, Y_{c}, Z_{c}\right\}$ such that the optical center $O_{c}$ corresponds to the robot's center $[x(t), z(t)]^{T}$ and the optical axis $Z_{c}$ is aligned with the robot's forward direction.

The main objective of this paper is to steer this nonholonomic vehicle through a door by using measurements coming from the on-board camera. The door is represented by two visual landmarks located on each of its two vertical supports, at the same height $h_{w}$ w.r.t. the plane of the robot motion. We denoted them by $F^{L}$ and $F^{R}$ (apices $R$ and $L$ indicate the "Right" and "Left" support of the door). Without loss of generality, we assume that the cartesian coordinates of these two points w.r.t. $\langle W\rangle$ are $F^{R}=\left(0, h_{w}, a\right)$ and $F^{L}=\left(0, h_{w},-a\right)$, respectively.

Based on the pinhole camera model [23], the position of the projection of the landmark in the image plane is given by

$$
\begin{aligned}
{ }^{I} x_{i} & =\alpha x \frac{{ }^{c} x_{i}}{{ }^{c} z_{i}}, \\
{ }^{I} y_{i} & =\alpha \alpha_{y} \frac{h}{{ }^{c} z_{i}},
\end{aligned}
$$

with $i=\{R, L\}$ and where ${ }^{c} x_{i}$ and ${ }^{c} z_{i}$ are the coordinates of the $i$-th landmark in the camera frame $\langle C\rangle, h$ is the height of the landmarks w.r.t the plane $X_{c} \times Z_{c}$, while $\alpha_{x}$ and $\alpha_{y}$ are the camera intrinsic parameters, achievable by a calibration procedure and representing the focal length of the camera in terms of pixel dimensions in the $x$ and $y$ direction respectively. Notice that, plane $X_{c} \times Z_{c}$ usually does not coincide with the 
plane $X_{W} \times Z_{W}$. Finally, the velocity of the landmark w.r.t. the camera reference frame due to vehicle movements is given by

$$
\begin{aligned}
{ }^{c} \dot{x}_{i} & =\omega^{c} z_{i} \\
{ }^{c} \dot{y}_{i} & =0 \\
{ }^{c} \dot{z}_{i} & =-v-\omega^{c} x_{i},
\end{aligned}
$$

with $i=\{R, L\}$.
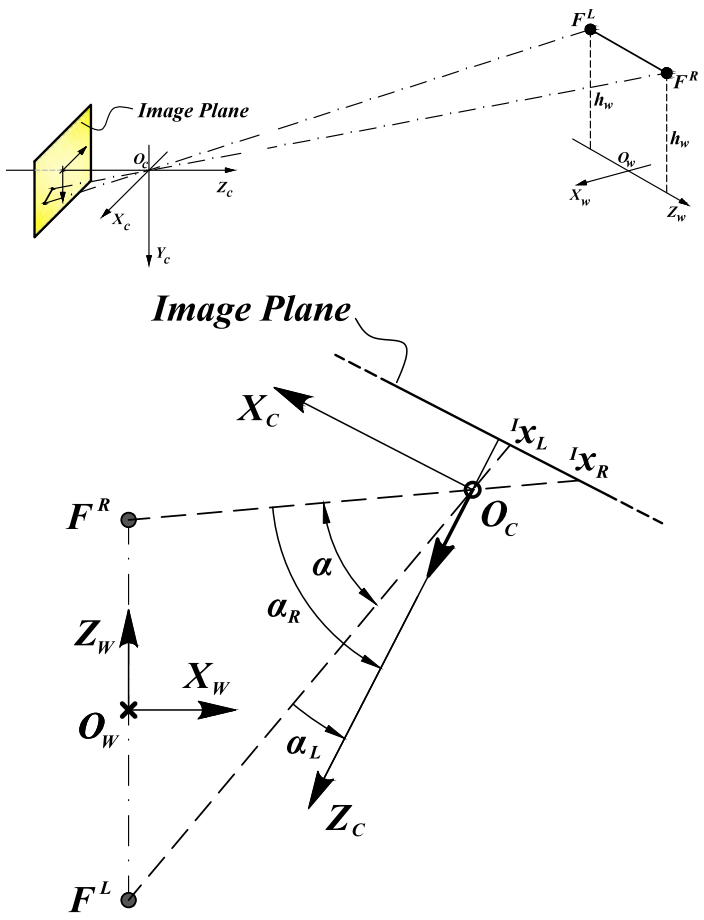

Fig. 1. Objective: to steer a vehicle through a door using only visual measures. The door is represented by two landmarks, $F^{L}$ and $F^{R}$ and the vehicle, represented as a directed point, has an on-board camera and is subject to nonholonomic constraints.

Remark 1: In next sections, we assume a camera with a large FOV so that the problem of keeping the landmarks in view is alleviated. This assumption does not impact the use of the control laws provided in next sections on a real system where the camera has a limited FOV. However, in the Appendix, the FOV limits in case of a fixed on-board camera will be taken into account and analyzed.

Remark 2: Even if the methodology used in this paper to design the control laws can be used also for different nonholonomic system, e.g. unicycle, car-like and trailers, the feedback control laws developed in next sections work only with the unicycle. However, the obtained trajectories can be directly used as reference trajectories for those nonholonomic vehicles as long as a tracking controller is provided.

\section{Some BASIC Geometry Around The Door}

In this section we describe the intrinsic geometry that naturally emerges around the door and we show how this geometry is useful to design a feedback control law that steers the vehicle through it.
Referring to Fig. 1, assume that the forward velocity of the vehicle is constant, e.g. $v=1$, and the angular velocity is such that

$$
{ }^{I} x_{R}(t) \equiv-{ }^{I} x_{L}(t),
$$

for all $t \in[0, T]$. In this case the vehicle is aligned with the bisector of angle $F^{L O_{c} F}$. In other words, the bearing angles 1 $\alpha_{R}(t)$ and $\alpha_{L}(t)$ w.r.t. each landmark have equal values but opposite signs. Indeed, from (2) and (5) we have

$$
\frac{{ }^{c} x_{R}}{{ }^{c} z_{z_{R}}} \equiv-\frac{{ }^{c} x_{L}}{{ }^{c} z_{L}} \quad \Rightarrow \quad \tan \alpha_{R}(t) \equiv-\tan \alpha_{L}(t)
$$

and hence $\alpha_{R}(t)=-\alpha_{L}(t)=\frac{\alpha(t)}{2}$. Moreover, by deriving the first equality in (6) and substituting (4) with $v=1$, we obtain the control $\omega$ that steers the vehicle along a path where $(6)$ is satisfied, i.e.

$$
\omega=-\frac{{ }^{c} x_{L}{ }^{c} z_{R}^{2}+{ }^{c} x_{R}{ }^{c} z_{L}^{2}}{{ }^{c} x_{R}{ }^{c} x_{L}{ }^{c} z_{R}^{2}+{ }^{c} x_{R}^{2} z_{L}^{2}+{ }^{c} z_{R}^{2}{ }^{c} z_{L}\left({ }^{c} z_{R}+{ }^{c} z_{L}\right)} .
$$

By using this strategy, the vehicle moves along a hyperbola, i.e. the locus of points where the absolute value of the difference between distances to the two foci (i.e. the projections on the motion plane of the two landmarks), is constant. Indeed, let us consider the distances between the robot and each landmark $\rho_{R}=\sqrt{{ }^{c} x_{R}^{2}+{ }^{c} z_{R}^{2}}$ and $\rho_{L}=\sqrt{{ }^{c} x_{L}^{2}+{ }^{c} z_{L}^{2}}$ and their dynamics. By using (4) with $i=R, L$, respectively, and by setting $v=1$, the dynamics of $\rho_{R}$ and $\rho_{L}$ reduces to

$$
\dot{\rho}_{R}=-\frac{z_{R}}{\rho_{R}}, \quad \dot{\rho}_{L}=-\frac{z_{L}}{\rho_{L}} .
$$

As $z_{R}=\rho_{R} \cos \alpha_{R}$ and $z_{L}=\rho_{L} \cos \alpha_{L}$, if the control $\omega$ given by (7) is applied, $\alpha_{R}=\alpha_{L}=\alpha / 2$ and hence

$$
\dot{\rho}_{R}-\dot{\rho}_{L}=-\cos \alpha_{R}+\cos \alpha_{L}=0 .
$$

We conclude that $\rho_{R}-\rho_{L}=K=$ const. along the path. Constant $K$ is equal to $2 a$, i.e. the distance between the two landmarks. The parametric equations of a generic hyperbola with foci $F^{R}$ and $F^{L}$ are given by

$$
\begin{aligned}
& x=a \cos \eta \sinh \xi \\
& z=a \sin \eta \cosh \xi
\end{aligned}
$$

with $\xi \in[0, \infty]$ and $\eta$ constant with values in $[-\pi, \pi)$. In the canonical form we have $\frac{z^{2}}{a^{2} \sin ^{2} \eta}-\frac{x^{2}}{a^{2} \cos ^{2} \eta}=\cosh ^{2} \xi-\sinh ^{2} \xi=$ 1 . Hence, curves with constant $\eta$ form hyperbolae. In the special case of $\eta \in\{0, \pm \pi\}$, the hyperbola degenerates into a straight line passing perpendicularly through the middle of the segment between $F^{R}$ and $F^{L}$ (see Fig. 2).

Notice that, by using the strategy described above, the vehicle definitely goes through the door. Indeed, any hyperbola crosses the segment between the landmarks. However, among all hyperbolae, the one followed by the vehicle depends on initial conditions. As a consequence, the vehicle might pass too near to the left or to the right jamb of the door. However,

\footnotetext{
${ }^{1}$ The bearing angle w.r.t. a goal is the angle between the heading of the robot and the direction to the goal.
} 
a good behavior would be to go as close as possible to the middle of the door. We will address this issue in section IV.

Coming back to parametric equations of the hyperbola $(8)$, let us consider the case in which $\xi$ is constant and $\eta$ varies. Such curves, which are known as ellipses, can be expressed in the canonical form as $\frac{x^{2}}{a^{2} \sinh ^{2} \xi}+\frac{z^{2}}{a^{2} \cosh ^{2} \xi}=\cos ^{2} \eta+\sin ^{2} \eta=1$.

The bundle of hyperbolae, obtained for different values of $\eta$ and the bundle of ellipses, obtained for different values of $\xi$, form an orthogonal coordinate system, a.k.a. elliptic coordinates, in which the coordinate lines are confocal ellipses and hyperbolae.

\section{A. Elliptic Coordinates}

Denoting by $\rho_{R}=\sqrt{(z-a)^{2}+x^{2}}$ and $\rho_{L}=\sqrt{(z+a)^{2}+x^{2}}$ the distance from the foci, i.e. the projections in the plane of the robot motion of the landmarks $F^{R}$ and $F^{L}$, respectively, the elliptic coordinates $(\xi, \eta)$ can be expressed as

$$
\xi=\operatorname{arccosh}\left(\frac{\rho_{R}+\rho_{L}}{2 a}\right), \quad \eta=\frac{\pi}{2}-\arccos \left(\frac{\rho_{L}-\rho_{R}}{2 a}\right)
$$

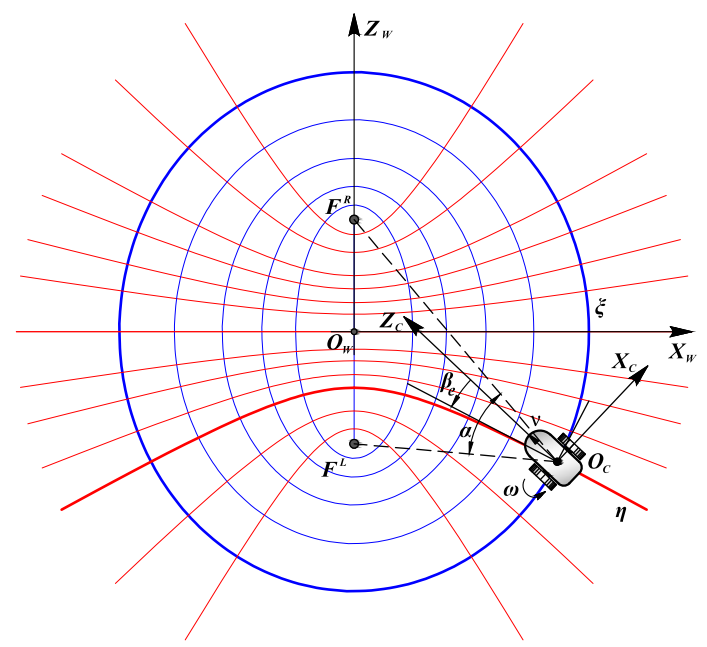

Fig. 2. Elliptic coordinate system. Ellipses and hyperbolae intersect perpendicularly.

Notice that, at the limit $a \rightarrow 0$, elliptic coordinates reduce to polar coordinates $(\rho, \psi)$. In particular, $\eta \rightarrow \psi$ and $a \cosh \xi \rightarrow \rho$. This also happens when the vehicle is sufficiently far from the door. To complete this set of coordinates and to univocally describe the vehicle configurations, let us introduce a generalization of the bearing angle that is the angle between the heading of the vehicle and the tangent to the hyperbola passing through the vehicle position:

$$
\beta_{e}=\arctan (\tanh \xi \tan \eta)-\theta+\pi .
$$

Remark 3: Assuming a calibrated camera, elliptic coordinates can be directly computed from the measurements of features in the image plane, hence basically without a state observer, even if $h$ is unknown. Indeed, from (2) and (3) we have

$$
\rho_{i}=\frac{\alpha_{y}}{\alpha_{x}} \frac{h}{I y_{i}} \sqrt{{ }^{I} x_{i}^{2}+\alpha_{x}^{2}}, \quad i=R, L .
$$

while the distance between the two landmarks is given by

$$
2 a=\frac{\alpha_{y} h}{\alpha_{x}^{I} y_{R}{ }^{I} y_{L}} \sqrt{\left({ }^{I} x_{R}{ }^{I} y_{L}-{ }^{I} x_{L}{ }^{I} y_{R}\right)^{2}+\alpha_{x}^{2}\left({ }^{I} y_{L}-{ }^{I} y_{R}\right)^{2}} .
$$

Hence,

$$
\begin{aligned}
& \xi=\operatorname{arccosh}\left(\frac{{ }^{I} y_{L} \sqrt{{ }^{I} x_{R}^{2}+\alpha_{x}^{2}}+{ }^{I} y_{R} \sqrt{{ }^{I} x_{L}^{2}+\alpha_{x}^{2}}}{\sqrt{\left({ }^{I} x_{R}{ }^{I} y_{L}-{ }^{I} x_{L}{ }^{I} y_{R}\right)^{2}+\alpha_{x}^{2}\left({ }^{I} y_{L}-{ }^{I} y_{R}\right)^{2}}}\right) \\
& \eta=\frac{\pi}{2}-\arccos \left(\frac{{ }^{I} y_{L} \sqrt{{ }^{I} x_{R}^{2}+\alpha_{x}^{2}}-{ }^{I} y_{R} \sqrt{{ }^{I} x_{L}^{2}+\alpha_{x}^{2}}}{\sqrt{\left({ }^{I} x_{R}{ }^{I} y_{L}-{ }^{I} x_{L}{ }^{I} y_{R}\right)^{2}+\alpha_{x}^{2}\left({ }^{I} y_{L}-{ }^{I} y_{R}\right)^{2}}}\right)
\end{aligned}
$$

that does not depend on $h$. Notice that, (11) and (12) come from the definition of the elliptic coordinates $(9)$. For geometric properties, $\rho_{R}+\rho_{L} \geq 2 a$ and $\rho_{R}-\rho_{L} \leq 2 a$ and hence, the arguments in (11) and (12) are always well defined. Moreover, for the particular 3D positions of landmarks, the denominators cannot be equal to zero.

Finally, it is easy to prove that the bearing angle $\beta_{e}$ is given, in the image plane, by

$$
\beta_{e}=-\frac{1}{2}\left(\arctan \left(\frac{{ }^{I} x_{R}}{\alpha_{x}}\right)+\arctan \left(\frac{{ }^{I} x_{L}}{\alpha_{x}}\right)\right)
$$

Notice that, in case of landmarks at different height, results in Remark 3 do not hold anymore, as coordinates $(\xi, \eta)$ depend also on those values. However, the heights are constant and can be considered known or estimated by an observer.

\section{B. Kinematic Model of the vehicle in Elliptic Coordinates}

The vehicle kinematic model in elliptic coordinates $\zeta=$ $\left(\xi, \eta, \beta_{e}\right)$, with $\xi \in[0, \infty]$ and $\eta \in(-\pi / 2, \pi / 2)$ is

$$
\begin{aligned}
\dot{\xi} & =-\frac{v \cos \beta_{e} \sec \eta \operatorname{sech} \xi}{a \sqrt{1+\tan ^{2} \eta \tanh ^{2} \xi}} \\
\dot{\eta} & =\frac{2 v \cos \eta \cosh \xi(\sin \gamma+\cos \gamma \tan \eta \tanh \xi)}{a(\cos (2 \eta)+\cosh (2 \xi))} \\
\dot{\beta}_{e} & =-\omega-\frac{2 v\left(\cos \beta_{e} \operatorname{sech} \xi \sin \eta-\sin \beta_{e} \sec \eta \sinh \xi\right)}{a(\cos (2 \eta)+\cosh (2 \xi)) \sqrt{1+\tan ^{2} \eta \tanh ^{2} \xi}}
\end{aligned}
$$

and $\gamma=\beta_{e}-\arctan (\tan \eta \tanh \xi)$. Notice that the denominators of previous equations is equal to zero if $\cos (2 \eta)+\cosh (2 \xi)=$ 0 . However, as $\cosh (2 \xi) \geq 1$, being exactly 1 when the vehicle is on the segment $\overline{F^{R} F^{L}}$, the expression can be zero only on $\overline{F^{R} F^{L}}$ if and only if $\eta= \pm \pi / 2$. Being $\eta= \pm \pi / 2$ only on the half-lines from $F^{R}$ and $F^{L}$ to infinity, i.e. along the $Z_{W}$ axis, outside the segment $\overline{F^{R} F^{L}}$, the denominators of the kinematic model in elliptic coordinates can never be zero.

Next section is dedicated to the design of a feedback control law that steers the vehicle through the middle of the door 
by exploiting the planar geometry that has been previously described.

Remark 4: The problem to be solved can be viewed as a stabilization problem in $\zeta=0$ by a smooth and time invariant feedback control law. However, the existence of such control law for this kind of nonholonomic systems is subject to the Brockett's result [24]. In the special case of (14), i.e. a driftless affine-in-control system, the input vector fields are linearly independent at the origin. Moreover, the number of controls is less than the number of state variables. Hence, we can conclude that there exists no solution to the stabilization problem by a smooth and time invariant feedback control law. Notice that, in polar coordinates, i.e. the degenerate case of elliptic coordinates in case of $a \rightarrow 0$, a solution to the stabilization problem at the origin exists, as proved in [25].

\section{FeEdBACK Control LaW in Elliptic COORDINATES}

Let us consider the problem of designing a feedback control law that steers the vehicle through the door as close as possible to the middle. To design a such control law, let us first consider the following change of inputs

$$
\begin{aligned}
& v=w a \cos \eta \cosh \xi \sqrt{1+\tan ^{2} \eta \tanh ^{2} \xi} \\
& \omega=-\omega_{o}+\frac{2 u(\cos \beta \operatorname{sech} \xi \sin \eta-\sin \beta \sec \eta \sinh \xi)}{a(\cos (2 \eta)+\cosh (2 \xi)) \sqrt{1+\tan ^{2} \eta \tanh ^{2} \xi}}
\end{aligned}
$$

where $w$ and $\omega_{o}$ are new control variables. By substituting (15) and (16) in (14) the kinematic model reduces to

$$
\begin{aligned}
\dot{\xi} & =-w \cos \beta_{e} \\
\dot{\eta} & =w \sin \beta_{e} \\
\dot{\beta}_{e} & =\omega_{o} .
\end{aligned}
$$

The objective is now to design $w$ and $\omega_{o}$ such that $\eta$ and $\beta_{e}$ converge to zero. Let us hence assume $w=\bar{w}$ and consider the following candidate of Lyapunov

$$
V\left(\eta, \beta_{e}\right)=\frac{1}{2}\left(\lambda \eta^{2}+\beta_{e}^{2}\right)
$$

where $\lambda$ is a positive constant parameter. Its time derivative, after substituting (17), $\dot{V}\left(\eta, \beta_{e}\right)=\bar{w} \lambda \eta \sin \beta_{e}+\beta_{e} \omega_{o}$, and by choosing

$$
\omega_{o}=-K \beta_{e}-\bar{w} \lambda \eta \frac{\sin \beta_{e}}{\beta_{e}}
$$

with $K>0$ a constant parameter, we obtain

$$
\dot{V}\left(\eta, \beta_{e}\right)=-K \beta_{e}^{2},
$$

that is negative semi-definite. However, the control $\omega_{o}$ is well definite and smooth everywhere. Let us define $R=$ $\left\{\left(\eta, \beta_{e}\right) \mid \dot{V}=0\right\}:$ in this case, we have that $R=\left\{\left(\eta, \beta_{e}\right) \mid \beta_{e}=\right.$ $0\}$, i.e. $\beta_{e}$ is constantly zero. As a consequence, in $R$, also $\dot{\beta}_{e}=0$. Hence, it is straightforward to observe that the only trajectory of (17) in $R$ with control input given by $(18)$ is such that $\dot{\beta}_{e}=0=\omega_{o}=-\bar{w} \lambda \eta$. By assuming that $\bar{w}$ and $\lambda$ are not zero, the previous equality is verified only if $\eta$ is equal to zero as well. In conclusion, $R$ does not contain any trajectory of the system, except the trivial trajectory $\left(\eta, \beta_{e}\right)=(0,0)$. All conditions of the local Krasowskii-Lasalle principle are satisfied. We hence conclude that every trajectory starting from inside a given level curve of $V$ that contains the origin, converges to the origin as $t \rightarrow \infty$. Moreover, as $V$ is radially unbounded, we can conclude on the global asymptotic stability of the origin. Of course, other nonlinear control approaches could be used with system (17) to steer the vehicle through the door. For example, an input-output feedback linearization with $\eta$ as measurement.

The control law (18), basically solves a path following problem in elliptic coordinates. A similar solution can be obtained in cartesian coordinates to stabilize the vehicle along the $Z_{W}$ axis (models (1) and (17) are very similar). However, elliptic coordinates have some advantages: they can be obtained directly from measurements in the image plane (hence basically a state observer is not required). Moreover, all hyperbolae pass through the door, hence guaranteeing that the vehicle goes through it.

Remark 5: Each hyperbola intersects each ellipse in two points, symmetric w.r.t. the $Z_{W}$ axis. The control laws developed in this paper are immune to this fact as long as in remarks 3 the order of the landmarks is respected: the landmark on the left side, labelled by "L", and the landmark on the right side, labelled by "R". On the other hand, by using this strategy, the vehicle cannot go through the door from the halfplane characterized by $x>0$ starting from the half-plane with $x<0$ and vice-versa. However, in a real scenario this is not a standard situation.

\section{THE BUNDLE OF CIRCLES}

The feedback control laws provided in previous section is not able to steer the vehicle through the middle of the door but only near to it. The distance between the middle of the door and the point where the vehicle crosses the door depends on both initial conditions and hence, the values of the constant parameters $K$ and $\lambda$ in (18) should be suitably chosen to reduce as much as possible this error.

In this section, we will provide a feedback control law able to drive the vehicle exactly to the middle of the door, independently from the initial configuration. We will start by analyzing the angle between the directions towards the two landmarks (angle $\alpha$ in Fig. 3) and its first time derivative. Then, we will show how this study brings to a particular geometry, i.e. two mutually orthogonal bundles of circles, that can be exploited to solve the problem at hand, overcoming all drawbacks of the control law furnished in the previous section.

For any point $O_{c}=(x, z)$ there always exists a circle $C_{\alpha}$ passing through $O_{c}$ and the projections of landmarks $F^{L}$ and $F^{R}$ in the motion plane (see Fig. 3). Angle $\alpha=\widehat{F^{L} O_{c} F}$ is constant along $C_{\alpha}$ (a.k.a. angle at the circumference).

Of course, on the contrary, for each value $\alpha \in[0, \pi]$ there are two circles passing through $F^{R}$ and $F^{L}$ and symmetric w.r.t. the $Z_{W}$ axis whose angle at the circumference is $\alpha$ : by varying $\alpha \in$ $[0, \pi]$ we obtain a bundle of circles $\mathscr{C}_{\alpha}$ that, with the previous 


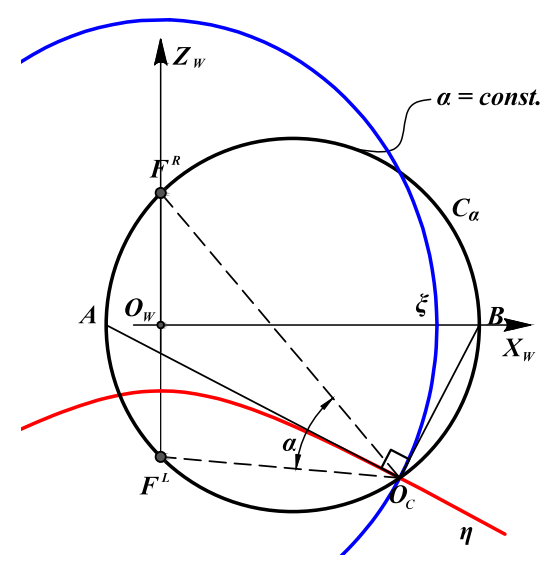

Fig. 3. For any point $O_{c}=(x, z)$ there always exists a circle $C_{\alpha}$ passing through $O_{c}$ and the projections in the motion plane of landmarks $F^{L}$ and $F^{R}$. The angle at the circumference $\alpha$ is constant along $C_{\alpha}$. Notice that, the tangent and perpendicular line in $O_{c}$ to the hyperbola through $O_{c}$, intersect the $X_{W}$ axis in points $A$ and $B$, respectively. The segment $\overline{A B}$ is the diameter of circle $C_{\alpha}$

defined bundle of hyperbolae, form a skew coordinates system. Indeed, circles and hyperbolae do not intersect orthogonally.

The expression of $\alpha$ in terms of $\xi$ and $\eta$ is

$$
\alpha=\arccos \left(1-\frac{4 \cos ^{2} \eta}{\cos (2 \eta)+\cosh (2 \xi)}\right) .
$$

while its time derivative $\dot{\alpha}$, which is not reported here for the sake of space, assumes the maximum value when

$$
\beta_{e}=\beta_{\max }=-\operatorname{sgn}(\eta) \arccos \left(\frac{\sqrt{2} \cos \eta \cosh \xi}{\sqrt{\cos (2 \eta)+\cosh (2 \xi)}}\right),
$$

hence necessarily $\beta_{e} \neq 0$. Indeed, if $\beta_{e} \equiv \beta_{\max }$ the vehicle is aligned to the perpendicular to the circle $C_{\alpha}$ passing through the current position of the vehicle. Of course, this happens for all values of $\alpha$ and for all points in $C_{\alpha}$. The set of all possible curves orthogonal to all members of $\mathscr{C}_{\alpha}$ constitutes a second bundle of circles $\mathscr{C}_{\alpha}^{\perp}$, as shown in Fig. 4. In other words, for any point $Q \in C_{\alpha}$ there always exists a circle $C_{\alpha}^{\perp}$ of $\mathscr{C}_{\alpha}^{\perp}$, perpendicular to $C_{\alpha}$ in $Q$. Moreover, $C_{\alpha}^{\perp}$ crosses perpendicularly all circles of $\mathscr{C}_{\alpha}$.

\section{A. Bipolar Coordinates}

The orthogonal bundles of circles previously introduced can be regarded as an orthogonal coordinates system also known as bipolar coordinates. The relationships between bipolar coordinates $\tau$ and $\alpha$ and the cartesian coordinates $x$ and $z$ are

$$
x=\frac{a \sin \alpha}{\cosh \tau-\cos \alpha}, \quad z=\frac{a \sinh \tau}{\cosh \tau-\cos \alpha},
$$

assuming poles $F^{R}$ and $F^{L}$ on the $Z_{W}$ axis. Moreover, $\tau$ and $\alpha$ assume values in the following ranges $-\infty \leq \tau \leq \infty$ and $0 \leq \alpha \leq \pi$.

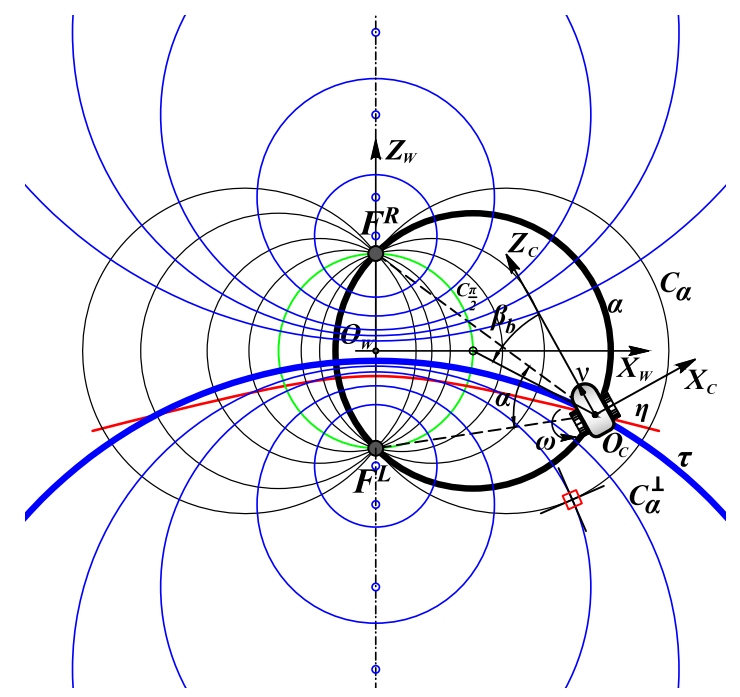

Fig. 4. The bipolar coordinate system consists of two orthogonal bundles of circles. Starting from the same point $O_{c}$, circle $C_{\alpha}^{\perp}$ crosses segment $\overline{F^{L} F^{R}}$ in a point which is closer to the middle of the door than the one reachable by following the hyperbola through $O_{c}$.

The bipolar coordinates expressed in terms of Cartesian ones are

$$
\begin{aligned}
\tau & =\log \left(\frac{\rho_{L}}{\rho_{R}}\right) \\
\alpha & =\arccos \left(\frac{\rho_{R}^{2}+\rho_{L}^{2}-4 a^{2}}{2 \rho_{R} \rho_{L}}\right) .
\end{aligned}
$$

From previous equations, after some algebra, it is possible to show that curves with constant $\tau$ are given by $x^{2}+$ $\left(z-\frac{a}{\tanh \tau}\right)^{2}=\frac{a^{2}}{\sinh ^{2} \tau}$, which is the equation of a circle whose center is on the $Z_{W}$ axis with coordinates $\left(0, \frac{a}{\sinh \tau}\right)$ and radius $R=\frac{a}{|\sinh \tau|}$. These circles have been previously denoted by $C_{\alpha}^{\perp} \in \mathscr{C}_{\alpha}^{\perp}$. On the other hand, if $\alpha$ is constant, we obtain curves given by $\left(x-\frac{a}{\tan \alpha}\right)^{2}+z^{2}=\frac{a^{2}}{\sin ^{2} \alpha}$ which is the equation of a circle passing through the projection in the motion plane of landmarks $F^{R}$ and $F^{L}$, centered on the $X_{W}$ axis at $\left(\frac{a}{\tan \alpha}, 0\right)$ and radius $R=\frac{a}{|\sin \alpha|}$. These circles have been previously denoted by $C_{\alpha} \in \mathscr{C}_{\alpha}$.

To describe the position of the vehicle in the motion plane w.r.t. the door, let us consider a slightly different pair of coordinates, i.e. $\tau$ and $\hat{\alpha}=\pi-\alpha$. Notice that, $\hat{\alpha}$ is the supplementary angle of the angle at the circumference. Moreover, the middle of the door is the origin of those coordinates. Finally, as for elliptic coordinates, to univocally describe the vehicle configuration, let us introduce the angle $\beta_{b}$ between the heading of the vehicle and the tangent to the circle $C_{\alpha}^{\perp}$ passing through the vehicle position. The expression of this angle w.r.t. $\alpha$ and $\tau$ and $\theta$ is

$$
\beta_{b}=\arctan \left(\frac{\sin \alpha \sinh \tau}{1-\cos \alpha \cosh \tau}\right)-\theta+\pi
$$

Remark 6: Assuming a calibrated camera, bipolar coordinates can be computed directly from the image plane 
measurements. Indeed, $\tau$ can be expressed in terms of image coordinates of the couple of features as $\tau=$ $\log \left(\frac{I_{y_{L}} \sqrt{{ }^{I} x_{R}^{2}+\alpha_{x}^{2}}}{I_{y_{R}} \sqrt{{ }^{I} x_{L}^{2}+\alpha_{x}^{2}}}\right)$. Moreover, $\hat{\alpha}$ can be easily obtained as $\hat{\alpha}=\pi-\left(\arctan \left(\frac{{ }^{I} x_{L}}{\alpha_{x}}\right)-\arctan \left(\frac{{ }^{I} x_{R}}{\alpha_{x}}\right)\right)$, while $\beta_{b}$ can be determined from (22), where $\theta$ can be obtained combining (10) with (13). Hence, also $\beta_{b}$ can be found directly from image plane measurements.

\section{B. Kinematic model of the vehicle in bipolar coordinates}

The kinematic model of the vehicle in bipolar coordinates $\lambda=\left(\tau, \hat{\alpha}, \beta_{b}\right)$ is

$$
\begin{aligned}
\dot{\tau} & =\frac{v}{a}\left((1+\cos \hat{\alpha} \cosh \tau) \sin \left(\beta_{b}-\operatorname{arccot}(\cot \hat{\alpha} \operatorname{coth} \tau+\csc \hat{\alpha} \operatorname{csch} \tau)\right)+\right. \\
& \left.+\cos \left(\beta_{b}-\operatorname{arccot}(\cot \hat{\alpha} \operatorname{coth} \tau+\csc \hat{\alpha} \operatorname{csch} \tau)\right) \sin \hat{\alpha} \sinh \tau\right) \\
\dot{\hat{\alpha}} & =\frac{v}{a}\left(-\cos \left(\beta_{b}-\operatorname{arccot}(\cot \hat{\alpha} \operatorname{coth} \tau+\csc \hat{\alpha} \operatorname{csch} \tau)\right)(\cos \hat{\alpha} \cosh \tau+1)+\right. \\
& \left.+\sin \hat{\alpha} \sin \left(\beta_{b}-\operatorname{arccot}(\cot \hat{\alpha} \operatorname{coth} \tau+\csc \hat{\alpha} \operatorname{csch} \tau)\right) \sinh \tau\right) \\
\dot{\beta}_{b} & =-\omega-\frac{v}{a} \frac{(\cos \hat{\alpha}+\cosh \tau)^{2}\left(\cos \beta_{b} \csc \hat{\alpha}+\operatorname{csch} \tau \sin \beta_{b}\right)}{(\cos \hat{\alpha}-\cosh \tau) \sqrt{1+(\cot \hat{\alpha} \operatorname{coth} \tau+\csc \hat{\alpha} \operatorname{csch} \tau)^{2}}} .
\end{aligned}
$$

Based on (19) and 20 the maximum value $\dot{\hat{\alpha}}_{\text {max }}$ of $\dot{\hat{\alpha}}$ is obtained along circles of $\mathscr{C}_{\alpha}^{\perp}$, i.e. with $\beta_{b}=0$. By the second of (23) we have

$$
\dot{\hat{\alpha}}_{\text {max }}=\left.\dot{\hat{\alpha}}\right|_{\beta_{b}=0}=-\frac{v(\cos \hat{\alpha}+\cosh \tau)}{a} .
$$

For positive values of $v$, function (24), is negative and is zero if $\tau=0$ and $\hat{\alpha}=\pi$ (or $\alpha=0$ ), i.e. very far from the door.

\section{A simple strategy to steer the vehicle through the door exploiting the geometric properties of the bundle of circles}

A strategy to steer the vehicle through the middle of the door follows from these geometric facts: for any point $Q \in C_{\alpha}^{\perp}$ the tangent line to $C_{\alpha}^{\perp}$ passes through the center of the circle $C_{\alpha}$ through $Q$. Moreover, the center of $C_{\alpha}$ is in turn at the intersection between axis $X_{W}$ and the circle $C_{2 \alpha}$ (i.e. a circle of $\mathscr{C}_{\alpha}$ whose angle at the circumference is $2 \alpha$ ). In particular, the center of circle $C_{\pi / 2}$ coincides with the middle of the door (see Fig. 4). Hence, starting from points $Q$ such that $\alpha<\pi / 2$, a possible strategy is to follow a circle $C_{\alpha}^{\perp}$ of bundle $\mathscr{C}_{\alpha}^{\perp}$ until the circumference characterized by $\alpha=\pi / 2$ is reached. Then, it is sufficient to move along a straight line to pass through the middle of the door.

Even if the strategy previously described is able to steer the vehicle through the middle of the door, it has some drawbacks w.r.t. the feedback control law given by (28) and (27). Indeed, while during the first phase a feedback control law guarantees that the vehicle follows a circle of $\mathscr{C}_{\alpha}^{\perp}$ until $C_{\pi / 2}$, during the second phase, a feedforward control with $\omega=0$ and $u=$ const . is applied. Moreover, the crossing angle, which is not $\pi / 2$, depends on the point reached on the circle $C_{\pi / 2}$. Finally, if at the beginning $\alpha>\pi / 2$, the vehicle has to reach $C_{\pi / 2}$ backward. Next section is dedicated to the design of a feedback control law in bipolar coordinates overcoming all drawbacks of the simple strategy described in this subsection.

\section{FEedBACK CONTROL LAW IN Bipolar COORDINATES}

The main idea of designing the feedback control law in bipolar coordinates is to define a smooth vector field $E(\cdot)$ obtained by derivation of an appropriate potential function $F(\cdot)$. Let us hence consider the following function

$$
F(\tau, \hat{\alpha})=\frac{(-\cos \hat{\alpha}+K \cosh \tau)}{a},
$$

where $K$ is a positive constant. This function is always positive and has global minimum at the middle of the door, i.e. with $\tau=$ 0 and $\hat{\alpha}=0$ (see also Fig. 5). Notice that, function $F(\cdot)$ is very similar to (24). Considering $F(\tau, \hat{\alpha})$ as a potential function,

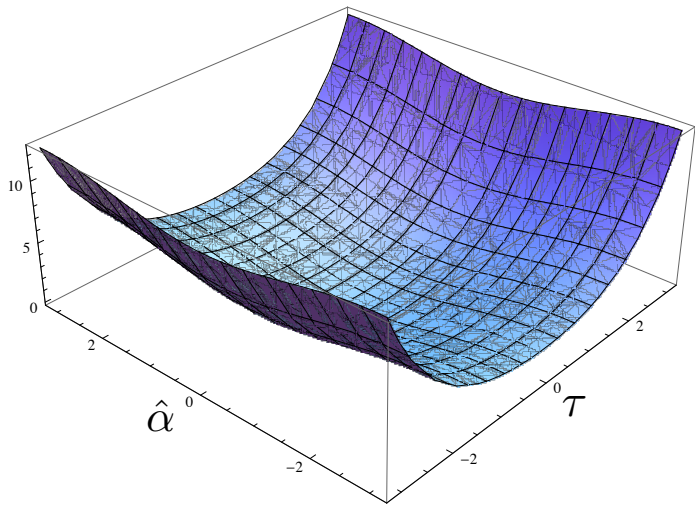

Fig. 5. Function $F(\tau, \hat{\alpha})$ for different values of $\hat{\alpha}$ and $\tau$ and $K=1$. The minimum is at the origin, i.e. at the middle of the door.

the associated vector field is

$$
E(\tau, \hat{\alpha})=\nabla F(\tau, \hat{\alpha})=\frac{\cosh \tau-\cos \hat{\alpha}}{a^{2}}\left[\begin{array}{c}
K \sinh \tau \\
-\sin \hat{\alpha}
\end{array}\right] .
$$

Fig. 6(a) shows level curves of $F(\tau, \hat{\alpha})$ as well as the vector field. Notice that, all flow lines converge toward point $(\tau, \hat{\alpha})=$ $(0,0)$, that, in Cartesian coordinates, corresponds to the middle of the door. In Fig. 6(b), the same potential field and associated vector field in Cartesian coordinates is also reported.

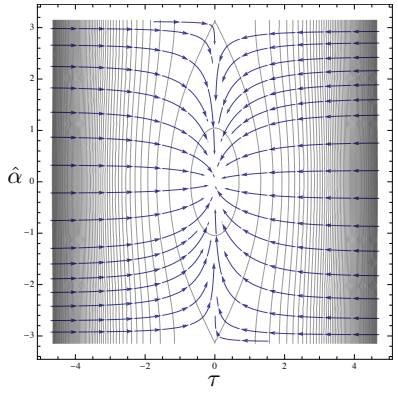

(a) Vector field $E(\tau, \hat{\alpha})$.

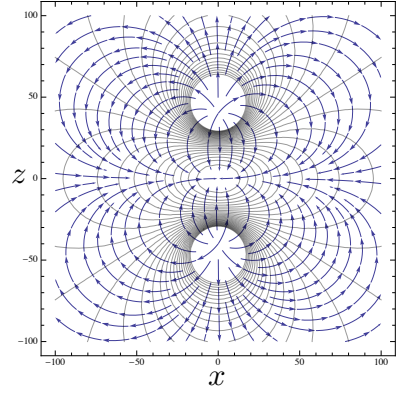

(b) Vector field $E(\tau, \hat{\alpha})$ expressed in Cartesian coordinates.
Fig. 6. Vector field obtained as the gradient of $F(\tau, \hat{\alpha})$ with $K=1$. 
The objective is now to determine a feedback control law that steers the vehicle along the vector field represented in Fig. 6(a) by means bipolar coordinates, or represented in Fig. 6(b) by means Cartesian ones. Let $\phi$ be the bearing angle $\beta_{b}$ when the vehicle is aligned with the vector field at any point $(\tau, \hat{\alpha})$. The angle $\phi$ can be easily obtained from (26),

$$
\phi=-\arctan 2(K \sinh \tau, \sin \hat{\alpha}) .
$$

Let us define the error $\sigma=\beta_{b}-\phi$ and force the dynamics of $\sigma$ to be $\dot{\sigma}=-K_{\beta} \sigma, K_{\beta}>0$, by

$$
\begin{aligned}
& \omega=K_{\beta}\left(\beta_{b}-\phi\right)+\dot{\phi}+ \\
& -\frac{v}{a} \frac{(\cos \hat{\alpha}+\cosh \tau)^{2}\left(\cos \beta_{b} \csc \hat{\alpha}+\operatorname{csch} \tau \sin \beta_{b}\right)}{(\cos \hat{\alpha}-\cosh \tau) \sqrt{1+(\cot \hat{\alpha} \operatorname{coth} \tau+\csc \hat{\alpha} \operatorname{csch} \tau)^{2}}} .
\end{aligned}
$$

Once the vehicle is aligned with the vector field, it should reach the middle of the door. To do that, let us consider the following continuously differentiable function $V$ in terms of $\tau$, $\hat{\alpha}$ and $\sigma$,

$$
V=\frac{1}{2}\left(\tau^{2}+\hat{\alpha}^{2}+\sigma^{2}\right),
$$

and consider its time derivative along the trajectories of the system. By using 27) we obtain

$$
\dot{V}=-K_{\beta}\left(\beta_{b}-\phi\right)^{2}-v \frac{(\cos \hat{\alpha}+\cosh \tau)\left(\hat{\alpha} \cos \beta_{b}-\tau \sin \beta_{b}\right)}{a} .
$$

and, by choosing the forward velocity as

$$
v=K_{v}\left(\hat{\alpha} \cos \beta_{b}-\tau \sin \beta_{b}\right),
$$

we finally have

$\dot{V}=-K_{1}\left(\beta_{b}-\phi\right)^{2}-\frac{(\cos \hat{\alpha}+\cosh \tau)\left(\hat{\alpha} \cos \beta_{b}-\tau \sin \beta_{b}\right)^{2}}{a} \leq 0$

which is negative semi-definite. As the function $V$ is positive definite, according to the Lasalle's invariance principle, the system trajectories converge to the largest invariant set $R=\{\lambda \mid \dot{V}(\lambda)=0\}$. By simple computation, $R=R_{1} \vee R_{2}$ where $R_{1}=\left\{\lambda \mid\left\{\hat{\alpha} \cos \beta_{b}-\tau \sin \beta_{b}=0\right\} \wedge\left\{\beta_{b}=\phi\right\}\right\}$ and $R_{2}=\left\{\lambda \mid\{\hat{\alpha}=0, \tau=0\} \wedge\left\{\beta_{e}=\phi\right\}\right\}$. After simple algebra, we obtain that $R_{1}=\{\lambda \mid\{\hat{\alpha}=\pi\} \wedge\{\tau=0\}\}$ while $R_{2}=\{\lambda \mid \lambda=0\}$. It is possible to show that, if $K \neq 0, R_{1}$ is not an invariant set. Indeed, for $\lambda=[0, \pi, 0]^{T}$ we have $v \neq 0$ and thus the system can escape from $R_{1}$. As a consequence, point $\lambda=(0,0,0)$ is the only invariant set and we can conclude on the local asymptotic stability of the origin. Moreover, as the Lyapunov function is radially unbounded, we can conclude on the global asymptotic stability of the origin. Differently from the control law developed in Section IV, the control law developed in this section is able to steer the vehicle exactly through the middle of the door.

As for hyperbolae and ellipses, each circle of bundle $C_{\alpha}$ intersects each circle of bundle $C_{\alpha}^{\perp}$ in two points, symmetric w.r.t. the $Z_{W}$ axis. Also the control laws in bipolar coordinates are immune to this fact by following the same reasoning as in Remark 5 .

\section{Simulations}

In this section, simulations showing the effectiveness of control laws proposed in Sections IV] and VI are presented. For both cases, two virtual scenario are considered. In the first one, the robot is inside a room and the objective is to leave the room passing through the door. In the second one, the robot is in a corridor and the objective is to enter a room passing through the door. The door is represented by two 3D points located at $(0,70,40) \mathrm{cm}$ and at $(0,70,-40) \mathrm{cm}$ w.r.t. a global reference frame. These points are at the same height above the plane of the robot motion. The 3D points of the scene are projected in the image plane through a simulated pinhole camera. The size of the image is $640 \times 480$ and the characteristic angle of the camera is almost $\pi$. Moreover, the image frames are captured with a frequency of 15 frames per second. If possible, both elliptic and bipolar coordinates are computed through image measurements and different level of white gaussian noise with standard deviation $\sigma=5,15$ pixel to points in the image plane is also added. Indeed, as in this paper the camera is fixed on the robot, it is not possible in the second scenario (see Figs. 7(c) and 8(c)) to perform the task without loosing at least one landmark, even if a very large FOV has been considered. For this reason, in the second scenario we directly assume that elliptic or bipolar coordinates are available and some white Gaussian noise, equivalent to have a 5 pixel Gaussian noise in the image plane, is directly added to the state variables.

In Figs. 7 and 8 trajectories of the vehicle are shown with and without noise for the two cases, chosen among the several trajectories analyzed for each case. The absolute average error w.r.t. the middle of the door is also reported in the caption of each picture with and without noise, i.e. $\varepsilon_{w}$ and $\varepsilon_{w o}$, respectively. The simulations confirm that both feedback control laws work properly in spite of image noise. Moreover, while the control law in elliptic coordinates is only able to steer the vehicle near the middle of the door (the error depends on the constant parameters in the control law), the one in bipolar coordinates can drive the vehicle exactly to the middle of the door. However, this is not the only difference. In particular, in bipolar coordinates the behavior of the vehicle is more suitable to accomplish the task of entering in a room from a corridor. On the other hand, both methods are able to accomplish the task of going out of a room even if the trajectories followed by the vehicle are quite different, being more suitable the one in elliptic coordinates. Indeed, in elliptic coordinates, small variations in the control parameters traduce in very different shape of the trajectories. However, to reduce as much as possible the crossing error at the entrance of the door, this parameters should be selected as a function of the initial conditions. It is important to note that both the control laws in elliptic and bipolar coordinates guarantee good performance with levels of image noise up to 15 pixels, which constitutes a very high amount of noise. The use of a feedback control expressed in elliptic and bipolar coordinates seems to play a very important role in reducing the effects of noise. Future works will be dedicated to deeply analyze this aspect. However, the control law in bipolar coordinates 

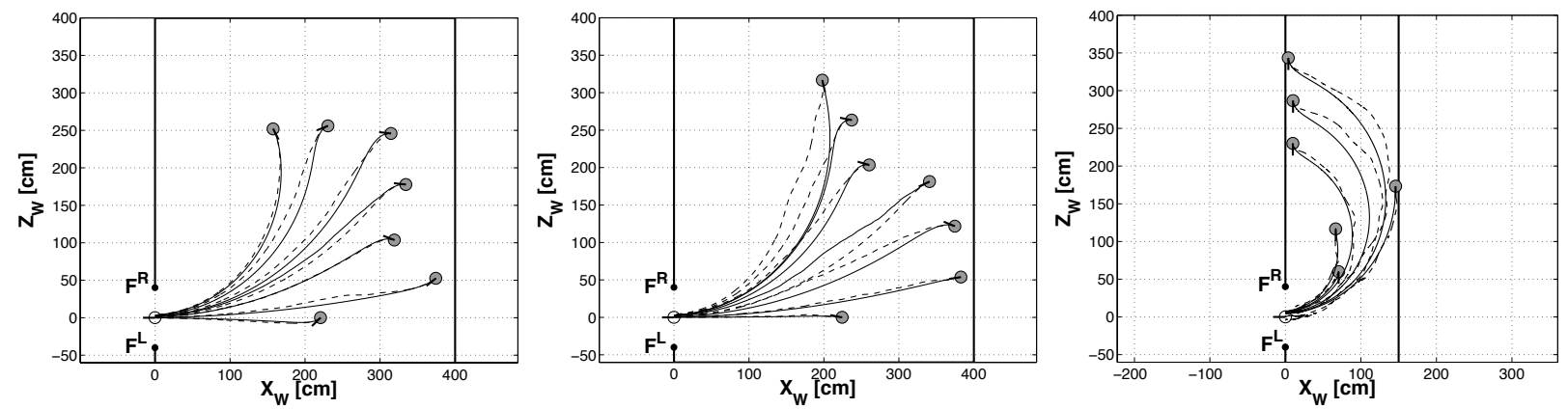

(a) $\sigma=5$ pixel: $\varepsilon_{w}=2.00 \pm 1.15 \mathrm{~cm}$ and $\varepsilon_{w o}=(b)$

(b) $\sigma=15$ pixel: $\varepsilon_{w}=2.61 \pm 1.43 \mathrm{~cm}$ and $1.79 \pm 1.02 \mathrm{~cm}$. $\varepsilon_{w o}=1.88 \pm 1.03 \mathrm{~cm}$.

$6.85 \pm 2.92 \mathrm{~cm}$.

Fig. 7. Simulations with the feedback control law in elliptic coordinates: trajectories of the vehicle without and with white gaussian noise, continuous and dashed lines, respectively. The vehicle leaves a room in (a), (b) and (c) and enters a room from a corridor (d), by passing through a door.

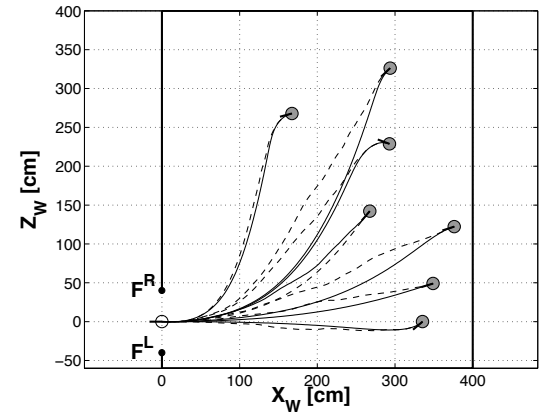

(a) $\sigma=5$ pixel: $\varepsilon_{v}=0.0028+0.096 \mathrm{~cm}$ and $($ b) $\varepsilon_{w o}=0.0017 \pm 0.0011 \mathrm{~cm}$.

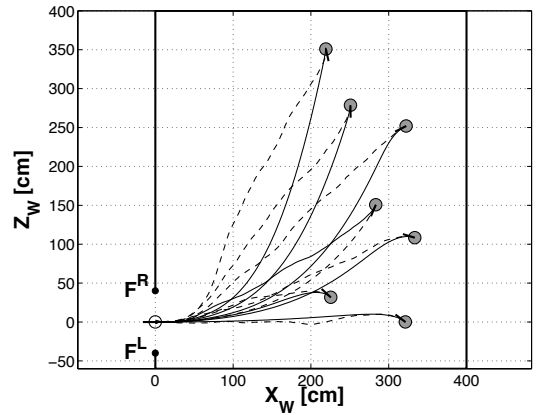

(b) $\sigma=15$ pixel: $\varepsilon_{w}=$

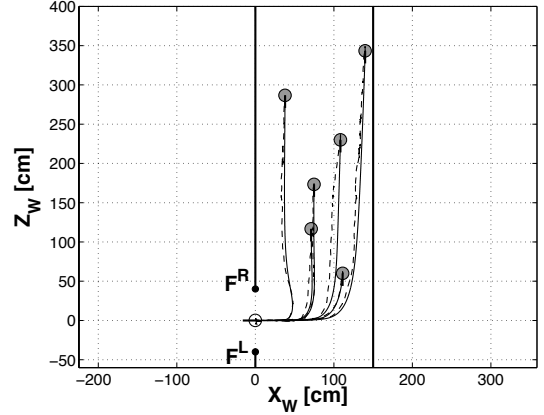

(c) $\sigma=5$ pixel: $\varepsilon_{w}=0.1 \pm 0.33 \mathrm{~cm}$ and $\varepsilon_{w o} \approx$ $0 \mathrm{~cm}$.

Fig. 8. Simulations with the feedback control law in bipolar coordinates: trajectories of the vehicle with and without white gaussian noise, continuous and dashed lines, respectively. The vehicle leaves a room in (a), (b) and (c) and enters a room from a corridor (d), by passing through a door.

seems to be more sensitive to image noise than the control law in elliptic coordinates when the vehicle is far away from the door, making difficult the tuning of the control parameters. On the other hand, the control laws in bipolar coordinates work very well near the door, especially while entering a room from the corridor. This may suggest a possible strategy: use elliptic control laws when the robot is far away from the door and bipolar ones when it is sufficiently near the door or when the vehicle is very near the wall.

As a final observation, it is important to point out that, even if the method relies on the assumption that the two landmarks are at the same height, we have simulated our method also in case of differences in height up to $10 \mathrm{~cm}$, observing that the method works quite well also in this case. For the sake of space, we have not reported these simulations.

Both control laws depend on parameter $a$ which is half of the width of the door. This parameter is constant and can be assumed to be known or estimated via a suitable observer. However, Fig. 9 shows how errors in the estimation of $a$ influence the trajectories of the vehicle. In particular, starting from the same configuration, we have considered three cases: $a=40 \mathrm{~cm}$ (the actual value), $a=80 \mathrm{~cm}$ (twice the actual value) and $a=20 \mathrm{~cm}$ (half of the actual value). Simulations show that errors in the estimation of parameter $a$ do not compromise the effectiveness of the control laws. The feedback control law in elliptic coordinates seems to be more sensitive to variations of the parameter $a$ than the one in bipolar coordinates.

\section{EXPERIMENTAL RESULTS}

Referring to Fig. 10, the experimental setup was comprised of a Vivotek Wireless Network Camera with PanTilt (PT7137) fixed at the middle of the wheel axel of the RobuLAB-10 (by Robosoft) mobile platform. The image resolution was $640 \times 480$ and the camera horizontal and vertical FOV amplitude are around $50 \mathrm{deg}$ and $40 \mathrm{deg}$, respectively. Moreover, in our experiments, the pan-tilt mechanism is not used. The camera has been calibrated by the Matlab ${ }^{\circledR}$ calibration toolbox. The result of this procedure, has furnished the focal length: $\alpha_{x}=712.28 \pm 3.32$ pixel and $\alpha_{y}=711.93 \pm$ 3.27 pixel; the principal point: $P_{c c}=[330.98,273.42] \pm$ $[6.69,6.06]$ pixel; the skew: $\alpha_{c}=0$, angle of pixel axes is $\pi / 2$; the distortion: $K_{c}=[-0.40,0.24,0.0018,-0.00011,0] \pm$ $[0.02,0.09,0.0015,0.0012,0]$; the pixel error: $p_{\text {err }}=$ $[0.35,0.29]$ pixel. Notice that, the camera presents a radial distortion. The noise level has been estimated to have a standard deviation $\sigma=0.05$ pixel and the controller rate was 


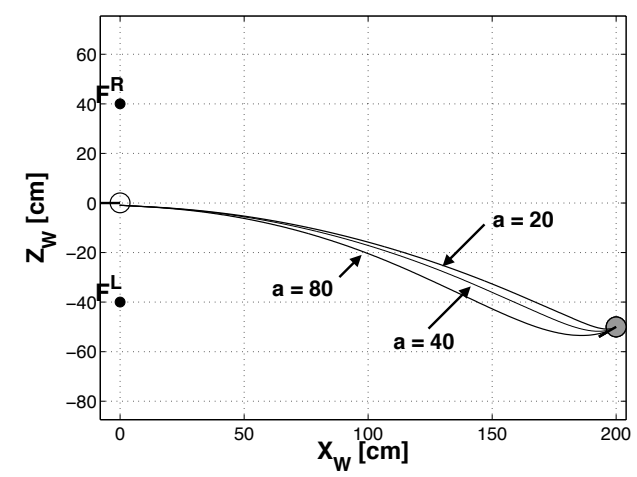

(a) Control laws in elliptic coordinates developed in section IV

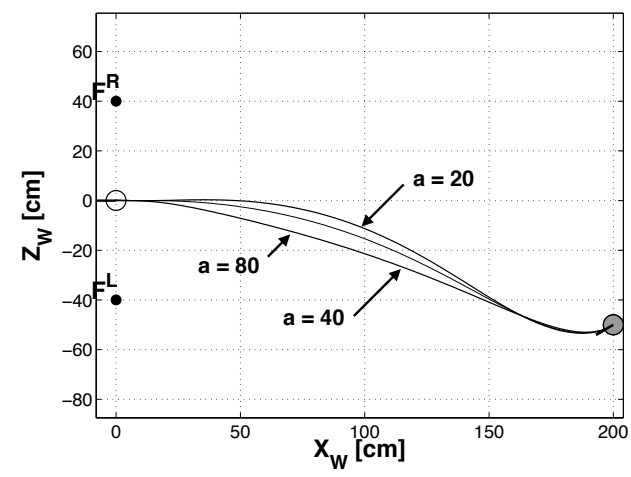

(b) Control laws in bipolar coordinates developed in section VI

Fig. 9. Trajectories of the vehicle starting from the same initial configuration $q=(200,-50,7 \pi / 6)$, but considering in the control law $a=40 \mathrm{~cm}$ (the actual value), $a=80 \mathrm{~cm}$ (twice the actual value) and $a=20 \mathrm{~cm}$ (half of the actual value).

around $20 \mathrm{~Hz}$. The distance between the two landmarks is $68 \mathrm{~cm}$.

Without loss of generality and to simplify the experimental setup, we have decided to avoid the use of door detection algorithms from which the two landmarks characterizing the door can be obtained. The door is indeed represented by two circular landmarks at the same height from the plane of the robot motion. The algorithms have been developed in $\mathrm{C}++$. Two scripts were running in parallel, communicating with each other by ROS Hydro. The first one was dedicated to grab an image from the wireless camera, filter it and detect the position of the landmarks in the image plane by using tools available in the OpenCV library. Once the landmarks are detected, we used functions provided by the VISP library to smoothly and robustly track the features in the image plane. The second script was dedicated to receive the landmarks position in the image plane, to use it to compute the control inputs $v$ and $\omega$ and to send them to the vehicle.

Because of the limited FOV aperture, the robot loses the landmarks while passing through the door. Indeed, as shown in the Appendix, there is a region around the door where the robot can not maintain in view the landmarks. To circumvent this problem, in our experiments, the parameters in the control laws are chosen in order to aligned the vehicle to the line passing through the middle of the door perpendicularly to the segment $\overrightarrow{F^{L} F^{R}}$, just before losing the landmarks. Starting from this configuration, which is quite near to the door, depending on the FOV amplitude, an open loop control law with $\omega=0$ and $v=$ const . is applied. Of course, other solutions might be used. For example, if the heights of the landmarks are available, by using the last measurements of the landmarks in the image plane (just before going outside) and the pinhole camera model, a prediction of the future positions of the landmarks in the image plane can be obtained. These predictions can be used in the feedback control laws provided in this paper to steer the vehicle through the door even if the landmarks are outside of the image plane. The heights of the landmarks can be assumed known or estimated by a suitable procedure or observer.

In Fig. 11 and Fig. 12 the trajectories of the robot starting from different initial configurations and obtained by applying the feedback control laws based on elliptic and bipolar coordinates, respectively, are reported. The state variables of the robot and hence the trajectories towards the door have been measured by using the Motion Analysis Capture system. Of course, no information coming from this sensor system are used in the control laws which provide a purely Image-Based visual servo control. A video of the experiments is available (Video).

\section{CONCLUSiOnS AND Future WORKS}

In this paper, a geometric approach to steer a robot subject to nonholonomic constraints through a door by using visual measures coming from a fixed on-board monocular camera, has been provided. The planar geometry that, in a natural way, has been built around the door consists of bundle of hyperbolae, ellipses and orthogonal circles. Moreover, feedback control laws and some strategies able to drive the vehicle through the door by exploiting this geometry have been established. Realistic simulations and experiments have been also reported to show the effectiveness of our solutions.

This work can be easily extended to other navigation and control problems, as e.g. controlling the vehicle amidst obstacles, under the condition that pairs of features that correspond to $3 \mathrm{D}$ points that in turns belong to different obstacles can be extracted from the image plane.

The vision-based strategy presented in this paper can also be related to human locomotion. In [26], by modeling the human locomotion as a unicycle ([27]), an inverse optimal control approach for understanding the cost functional that humans minimize during a rest-to-rest task, as e.g. to approach a door, has been used. The results enlighten the role of the bearing angle, and by consequence the role of vision, in the formation of locomotor trajectories. In Fig. 13(c) some examples of trajectories followed by humans are reported. Subjects, starting from the same initial configuration, have to reach different final positions with the same orientation, i.e. $120 \mathrm{deg}$ in Fig. 13(c) A similar experiment has been done here simulating the unicycle vehicle controlled by the feedback control laws in elliptic and bipolar coordinates developed in 

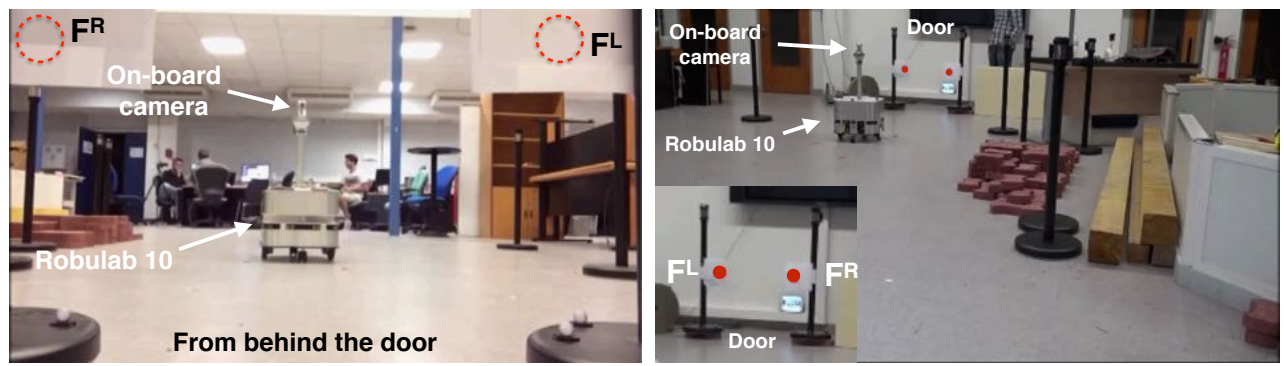

Fig. 10. Experimental setup.

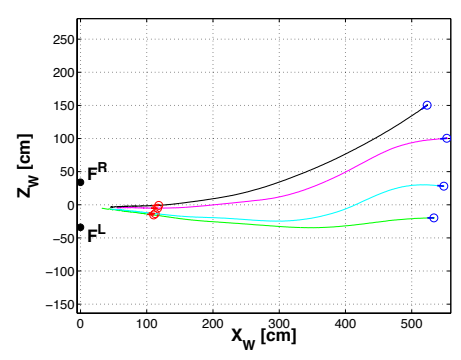

(a) Trajectories followed by the vehicle.

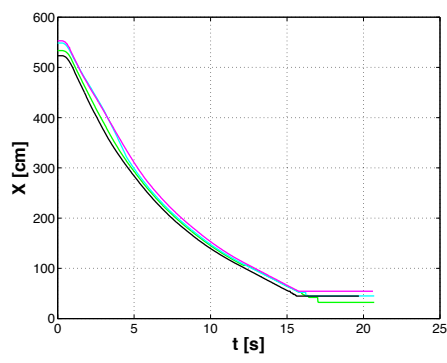

(b) $x$.

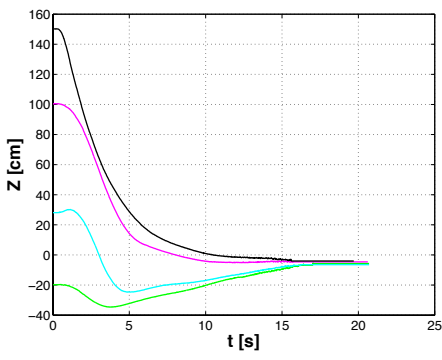

(c) $z$

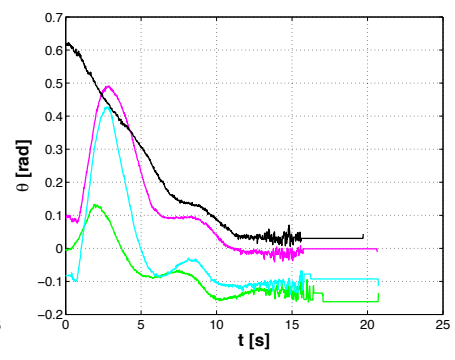

(d) $\theta$.

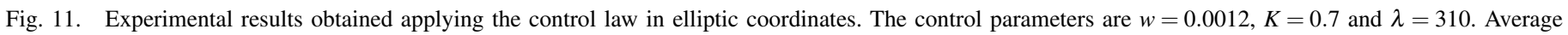
linear velocity of $0.3 \mathrm{~m} / \mathrm{s}$.

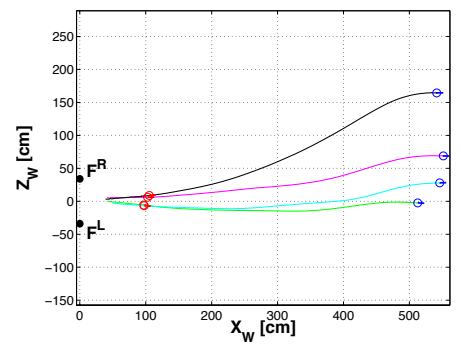

(a) Trajectories followed by the vehicle.

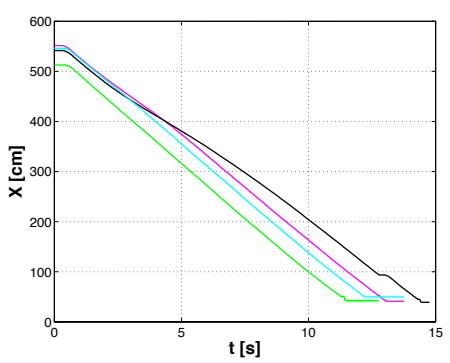

(b) $x$.

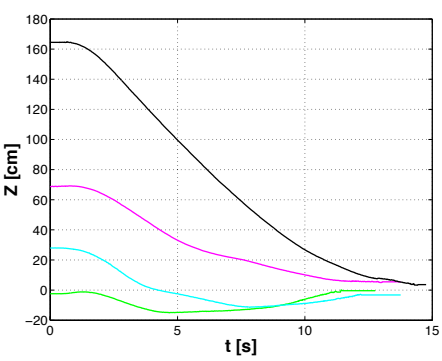

(c) $z$

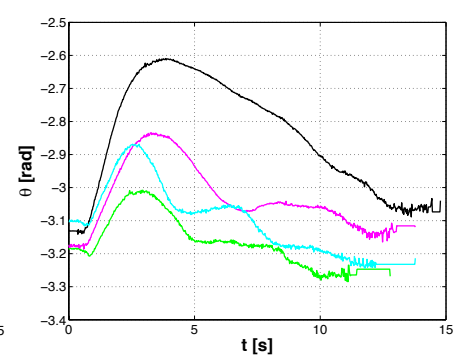

(d) $\theta$.

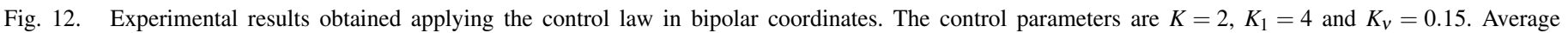
linear velocity of $0.4 \mathrm{~m} / \mathrm{s}$.

this paper. Results of this simulation are reported in Figs. 13(a) and 13(b) Even if the trajectories followed by the vehicle resemble the human ones, the choice of the values of the constant parameters that appear in the control laws are chosen by hand after several trials. Future works will be dedicated to better determine these values, and to introduce a pan-tilt mechanism to take into account that humans can turn the head and gaze [28]. However, when a human subject goes toward a target at a constant velocity it is shown that it behaves as a nonholonomic system: its velocity remains tangent to its sagittal plane $([27])$.

For all these reasons, it would be interesting to integrate this methodology in the classical walking pattern generators to steer humanoid robots, e.g. the HRP-2, towards a target, especially if, after a suitable analysis, the trajectories generated by using the control laws developed in this paper turn out to really be human-like. In this perspective, we will take also into account other biological results on how human use their visual sensor system in locomotion. In particular, we will study how our geometry allows to combine both the bearing angle and the focus of expansion to generate a visual feedback control of locomotion as humans do [29].

\section{REFERENCES}

[1] D. Dedieu, V. Cadenat, and P. Soueres, "Mixed camera-laser based control for mobile robot navigation," in IEEE/RSJ International Conference on Intelligent RObots and Systems, (IROS)., vol. 2, 2000, pp. 1081-1086.

[2] J.-B. Hayet, C. Esteves, G. Arechavaleta, O. Stasse, and E. Yoshida, "Humanoid locomotion planning for visually guided tasks," International Journal of Humanoid Robotics, vol. 09, no. 02, 2012. 

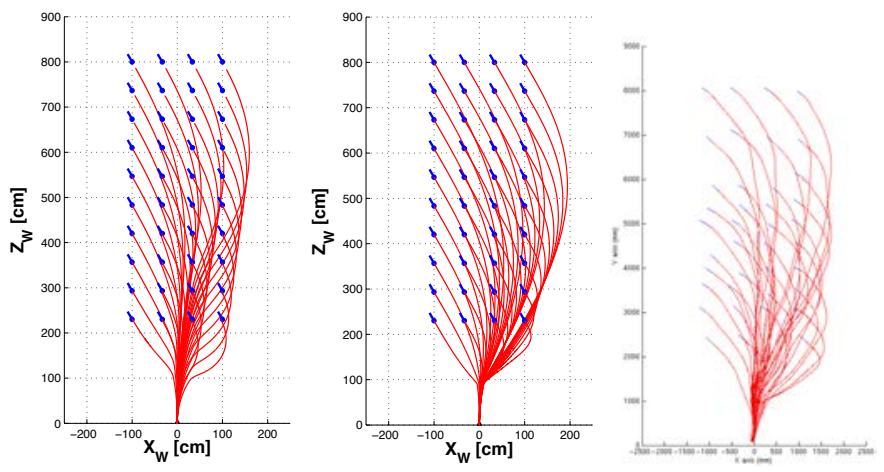

(a) By elliptic coordi- (b) By bipolar coordi- (c) By humans (From nates. nates. thors).

Fig. 13. Some examples of trajectories with the same final orientation, generated by the control laws in elliptic and bipolar coordinates and by Humans. In (d), (e) and (f) the final orientation id $120 \mathrm{deg}$.

[3] P. Salaris, D. Fontanelli, L. Pallottino, and A. Bicchi, "Shortest paths for a robot with nonholonomic and field-of-view constraints," IEEE Transactions on Robotics, vol. 26, no. 2, pp. 269-281, April 2010.

[4] S. Bhattacharya, R. Murrieta-Cid, and S. Hutchinson, "Optimal paths for landmark-based navigation by differential-drive vehicles with fieldof-view constraints," IEEE Transactions on Robotics, vol. 23, no. 1, pp. 47-59, February 2007.

[5] P. Salaris, L. Pallottino, S. Hutchinson, and A. Bicchi, "From optimal planning to visual servoing with limited fov," in Intelligent Robots and Systems (IROS), 2011 IEEE/RSJ International Conference on, Sept 2011, pp. 2817-2824.

[6] G. Lopez-Nicolas, N. Gans, S. Bhattacharya, C. Sagues, J. Guerrero, and S. Hutchinson, "Homography-based control scheme for mobile robots with nonholonomic and field-of-view constraints," IEEE Transactions on Systems, Man, and Cybernetics, Part B: Cybernetics, vol. 40, no. 4, pp. 1115-1127, Aug 2010

[7] P. Souéres, S. Tarbouriech, and B. Gao, "A robust vision-based controller for mobile robots navigation: application to the task sequencing problem," in IEEE/RSJ International Conference on Intelligent RObots and Systems (IROS), Aug 2005, pp. 2191-2196.

[8] R. F. Vassallo, H. J. Schneebeli, and J. Santos-Victor, "Visual navigation: combining visual servoing and appearance based methods," in International Symposium on Intelligent Robotic Systems (SIRS), vol. 98, 1998.

[9] R. Carelli, C. Soria, O. Nasisi, and R. Freire, "Stable agv corridor navigation with fused vision-based control signals," in IEEE 28th Conference of the Industrial Electronics Society (IECON), vol. 3, Nov 2002, pp. 2433-2438.

[10] A. Dev, B. Krose, and F. Groen, "Navigation of a mobile robot on the temporal development of the optic flow," in IEEE/RSJ International Conference on Intelligent Robots and Systems (IROS)., vol. 2, Sep 1997, pp. 558-563.

[11] F. Pasteau, V. K. Narayanan, M. Babel, and F. Chaumette, "A visual servoing approach for autonomous corridor following and doorway passing in a wheelchair," Robotics and Autonomous Systems, 2014, in press.

[12] I. Monasterio, E. Lazkano, I. Rañó, and B. Sierra, "Learning to traverse doors using visual information," Mathematics and Computers in Simulation, vol. 60, no. 3, pp. 347-356, 2002.

[13] A. Dev, B. Krose, and F. Groen, "Detecting, locating and crossing a door for a wide indoor surveillance robot," in IEEE International Conference on Robotics and Biomimetics (ROBIO)., Dec 2013, pp. 1740-1746.

[14] S. Patel, S.-H. Jung, J. Ostrowski, R. Rao, and C. Taylor, "Sensor based door navigation for a nonholonomic vehicle," in IEEE International Conference on Robotics and Automation (ICRA)., vol. 3, 2002, pp. 3081-3086.

[15] S. Wang, L. Chen, H. Hu, and K. McDonald-Maier, "Doorway passing of an intelligent wheelchair by dynamically generating bèzier curve trajectory," in IEEE International Conference on Robotics and Biomimetics (ROBIO), Dec 2012, pp. 1206-1211.

[16] C. Fernando A. Auat, D. L. C. Celso, B. Teodiano F., and C. Ricardo, "Slam-based cross-a-door solution approach for a robotic wheelchair," International Journal of Advanced Robotic Systems, vol. 7, no. 2, pp. 155-164, 2010.

[17] F. Cheein, C. De La Cruz, R. Carelli, and T. Bastos-Filho, "Solution to a door crossing problem for an autonomous wheelchair," in IEEE/RSJ International Conference on Intelligent Robots and Systems, Oct 2009, pp. 4931-4936.

[18] F. Chaumette and S. Hutchinson, "Visual servo control, Part I: Basic approaches," IEEE Robotics and Automation Magazine, vol. 13, no. 4, pp. 82-90, December 2006.

[19] — - "Visual servo control, Part II: Advanced approaches," IEEE Robotics and Automation Magazine, vol. 14, no. 1, pp. 109-118, March 2007.

[20] S. Hutchinson, G. D. Hager, and P. I. Corke, "A tutorial on visual servo control," IEEE Transactions on Robotics and Automation, vol. 12, no. 5, pp. 651-670, October 1996.

[21] P. Salaris, C. Vassallo, P. Souères, and J.-P. Laumond, "Geometry-based visual feedback control to go through a door," in IEEE International Conference on Robotics and Automation (ICRA), 2015, in press.

[22] J. Minguez, F. Lamiraux, and J.-P. Laumond, "Motion planning and obstacle avoidance," in Springer Handbook of Robotics, B. Siciliano and O. Khatib, Eds. Springer Berlin Heidelberg, 2008, pp. 827-852.

[23] R. Hartley and A. Zisserman, Multiple View Geometry in Computer Vision. Cambridge University Press, 2003.

[24] R. W. Brockett, "Asymptotic stability and feedback stabilization," in Differential Geometric Control Theory. Birkhauser, 1983, pp. 181191.

[25] M. Aicardi, G. Casalino, A. Bicchi, and A. Balestrino, "Closed loop steering of unicycle like vehicles via lyapunov techniques," Robotics Automation Magazine, IEEE, vol. 2, no. 1, pp. 27-35, Mar 1995.

[26] K. Mombaur, A. Truong, and J.-P. Laumond, "From human to humanoid locomotion-an inverse optimal control approach," Auton. Robots, vol. 28, no. 3, pp. 369-383, Apr 2010.

[27] G. Arechavaleta, J.-P. Laumond, H. Hicheur, and A. Berthoz, "On the nonholonomic nature of human locomotion," Autonomous Robots, vol. 25, no. 1-2, pp. 25-35, 2008.

[28] H. Hicheur, S. Vieilledent, and A. Berthoz, "Head motion in humans alternating between straight and curved walking path: Combination of stabilizing and anticipatory orienting mechanisms," Neuroscience Letters, vol. 383, pp. 87-92, 2005.

[29] W. H. Warren, B. A. Kay, W. D. Zosh, A. P. Duchon, and S. Sahuc, "Optic flow is used to control human walking," Nature neuroscience, vol. 4, no. 2, pp. 213-216, 2001.

[30] P. Salaris, A. Cristofaro, L. Pallottino, and A. Bicchi, "Epsilonoptimal synthesis for vehicles with vertically bounded field-of-view," IEEE Transactions on Control, 2014, accepted.

\section{APPENDIX}

The control laws developed in this paper do not take into account the Field-Of-View (FOV) limits of the on-board camera. In this appendix we will study and analyze this aspect. 


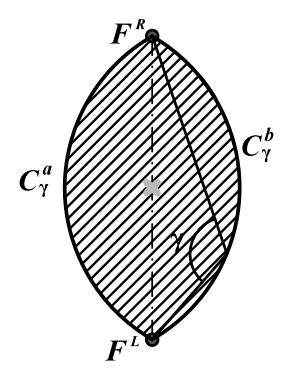

(a) Horizontal FOV limits.

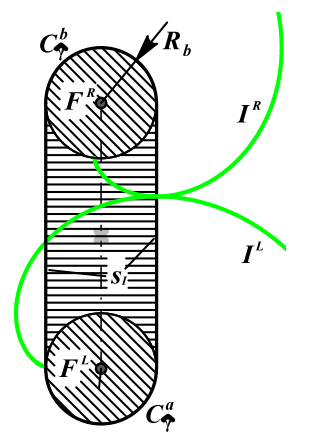

(b) Vertical FOV limits.

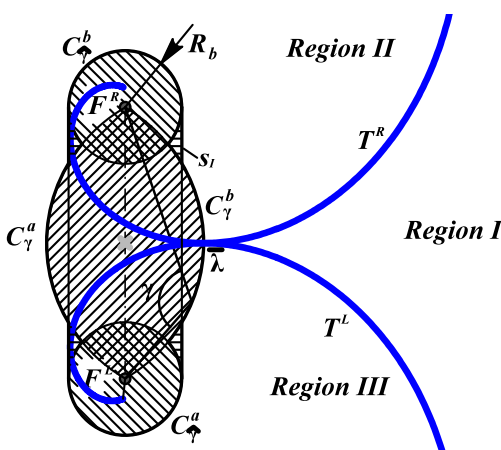

(c) Subdivision of the motion plane in regions according to the FOV limits in case of $\gamma<$ $2 \arcsin \left(R_{b} / a\right)$

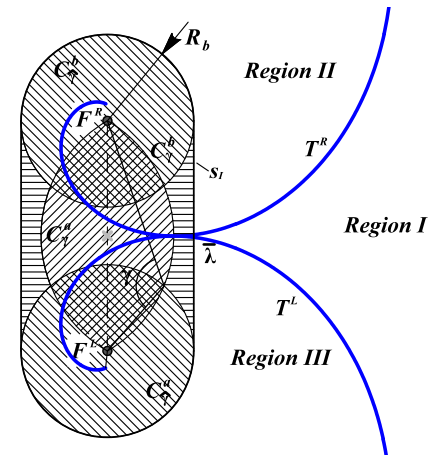

(d) Subdivision of the motion plane in case of $\gamma \geq 2 \arcsin \left(R_{b} / a\right)$.

Fig. 14. Analysis of the FOV limits.

\section{A. Analysis of FOV limits}

Let us first consider the region of points that violate the FOV limits for any orientation of the robot. In other words, in this region, both landmarks can not be maintained inside the FOV, whatever the orientation of the vehicle.

Let $\gamma$ and $\hat{\gamma}$ be the horizontal and vertical FOV aperture, respectively. Because of horizontal FOV limits, there exists a region $Z_{1}$ around the door such that the two landmarks can not be contemporary maintained between the left and right bounds of the image plane. It is straightforward to show (see [3]) that $Z_{1}$ is bounded by two arc of circles $\left(C_{\gamma}^{a}\right.$ and $\left.C_{\gamma}^{b}\right)$ whose angle at the circumference is exactly equal to $\gamma$ (see Fig. 14(a)).

Because of vertical FOV limits, considering each landmark separately, there is a region around it that the vehicle can not reach while maintaining the landmark between the upper and lower bounds of the image plane. Also in this case, it is easy to show (see [30]) that this regions is bounded by two circumferences $\left(C_{\hat{\gamma}}^{R}\right.$ and $C_{\hat{\gamma}}^{L}$ for the right and left landmark) centered at the projection of the landmark on the motion plane and with radius equal to $R_{b}=\frac{h}{\tan (\hat{\gamma} / 2)}$ (see Fig. 14(b)). However, if we consider the problem of maintaining both landmarks between the upper and lower bounds of the image pane, the region $Z_{2}$ that the vehicle can not reach is a planar capsule delimited by two straight line $s_{I}$ and two semicircle ends (see Fig. 14(b)). This is a direct consequence of the fact that when the landmark is on the upper (or lower) bound of the image plane, the vehicle is aligned with the tangent to the involute of circle passing through the current position of the vehicle (see [30]). These involute of circles, named by $I^{R}$ and $I^{L}$ (see Fig. 14(b), have $C_{\hat{\gamma}}^{R}$ and $C_{\hat{\gamma}}^{L}$, respectively, as base circles. When both features are on the upper (or lower) vertical border of the image plane, the vehicle is aligned with the tangent line to both involutes $I^{R}$ and $I^{L}$. Moreover, by geometrical construction, the perpendicular line to each involute in any point of it, is tangent to the base circle.

Considering now horizontal and vertical FOV limits together: the region of point that the vehicle can not reach while maintaining both landmark inside the image plane is
$Z_{1} \cup Z_{2}$. In Fig. 14(c) the subdivision of the motion plane based on previous analysis is shown in case of $\gamma<2 \arcsin \left(R_{b} / a\right)$, i.e. along the $X_{W}$ axis, the horizontal FOV limits are activated before the vertical ones while approaching the door.

\section{B. Control strategy in case of FOV limits}

From previous section, it is clear that the vehicle can not reach the middle of the door maintaining both landmarks in sight. To avoid this problem, the control law developed in Section $\overline{\mathrm{VI}}$ can be modified in order to reach the configuration $\bar{\lambda}=[0, \pi-\gamma, 0]^{T}$, i.e. point $\bar{\lambda}$ in Fig. $14(\mathrm{c})$, instead of the configuration $\bar{\lambda}=[0, \pi, 0]^{T}$. This can be done by considering the modified potential function

$$
F_{\gamma}(\hat{\alpha}, \tau)=\frac{(-\cos (\hat{\alpha}-(\pi-\delta))+K \cosh \tau)}{a}
$$

and vector field

$$
E_{\gamma}(\tau, \hat{\alpha})=\frac{\cosh \tau-\cos (\hat{\alpha}-(\pi-\delta))}{a}\left[\begin{array}{c}
K \sinh \tau \\
-\sin (\hat{\alpha}-(\pi-\delta))
\end{array}\right] .
$$

and finally the following candidate Lyapunov function

$$
V=\frac{1}{2}\left(\tau^{2}+(\hat{\alpha}-(\pi-\delta))^{2}+\sigma^{2}\right),
$$

where $\delta=\gamma$. Once the vehicle reaches $\bar{\lambda}$, an open loop control can be applied, e.g. with controls $v=$ const. and $\omega=0$. However, especially from a practical point of view, depending on both the value of $\gamma$ (and hence the distance between the reached point and the door) and the final error which depends also in the image noise, this strategy might steer the vehicle very far from the middle of the door. In this case, an estimation of the landmark positions based on the camera model can be used until a new pair of landmarks is available. To do that, the height of the landmarks must be known, e.g. by an observer that estimate $h$ during the first phase in which the vehicle is approaching $\bar{\lambda}$. Once $h$ is obtained, it can be used to estimate landmark positions on the image plane (even if their are outside it) that can be in turn used in the feedback control laws. 
Finally, when the vehicle is sufficiently far away from the door, the horizontal limits of the FOV become more restrictive than the vertical ones. From results reported in [3], when the landmark moves on the right or on the left border of the image plane, the vehicle moves along a logarithmic spiral rotating counterclockwise or clockwise, respectively, around the projection on the motion plane of the landmark position. Depending on the value of $\gamma$, there exist two logarithmic spirals, $T^{R}$ and $T^{L}$ in Fig. $14(\mathrm{c})$ passing through $\bar{\lambda}$, whose characteristic angle ${ }^{2}$ is constant and equal to $\gamma / 2$. The spirals are tangent in $(\tau, \pi-\gamma)$ and subdivide the motion plane in three regions. Referring to Fig. 14(c), starting from Region II and III with an orientation such that both landmarks are in view, the vehicle can not reach point $\bar{\lambda}$ maintaining both landmarks inside the FOV along the whole trajectory. From these regions, a preliminary maneuver that steers the vehicle in Region I is needed to achieve the task. Indeed, from Region I the control law obtained from (29) might be able to steer the vehicle in $\bar{\lambda}$ by appropriately choosing the control parameters. If one feature reaches the right or left border of the image plane, the vehicle can moves along the logarithmic spiral until point $\bar{\lambda}$ is reached. In this case, the value of $\delta$ to be used in the Lyapunov function (29) is $2 \arcsin \left(R_{b} / a\right)$.

Previous analysis is valid even if $R_{b} \geq a \sin (\gamma / 2)$, or $\gamma \geq$ $2 \arcsin \left(R_{b} / a\right)$. In this case, point $\bar{\lambda}$ is in the middle of the line $s_{I}$ where logarithmic spiral are not tangent (see Fig. 14(d)].

In particular, if $\gamma=\pi, C_{\gamma}^{a}$ and $C_{\gamma}^{b}$ reduce to the segment $\overline{F^{R} F^{L}}$ while the logarithmic spirals become two circles centered at the projection on the motion plane of each landmark and passing through the middle of the door.

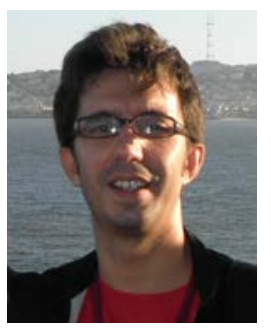

Paolo Salaris received the "Laurea" in Electrical Engineering in 2007 and the Doctoral degree in Robotics, Automation and Bioengineering in 2011 at the Research Center "E.Piaggio", University of Pisa. He has been Visiting Scholar at Beckman Institute for Advanced Science and Technology, University of Illinois, Urbana-Champaign in 2009. He has been a PostDoc at the Research Center "E.Piaggio" from 2011 to 2013 and currently is a PostDoc at LAASCNRS in Toulouse. His main research interests within Robotics are in optimal motion planning and control, control for nonholonomic systems, visual servoing and motion generation for humanoid robots.

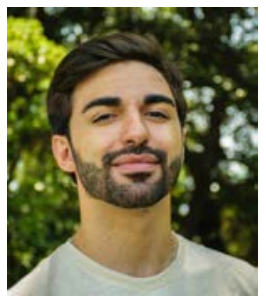

Christian Vassallo received his B.Sc. degree in Computer Engineering and M.Sc. in Robotics Engineering from the University of Genoa, Italy, in 2011 and 2013, respectively. He is currently working toward the Ph.D. degree in Robotics at LAAS-CNRS in Toulouse, France. His current research interests within humanoid robots include the analysis of human motion recorded by the motion capture system in order to extract rules, principles, and strategies that will be transfer to humanoid robots. The final goal of his research activity is to improve the humanoid robots capabilities to walk inspired to human ones.

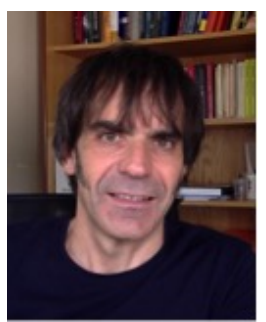

Philippe Souères is Director of Research at LAASCNRS in Toulouse. He received the M.S degree in Mathematics, the PhD in Robotics and the Habilitation from University Paul Sabatier of Toulouse in 1990, 1993 and 2001 respectively. From 1993 to 1994 he held a postdoctoral position at the University of California, at Berkeley in the EECS dept, under direction of Professor Shankar S. Sastry. Since that date he has been working on different facets of robot perception and control at LAAS-CNRS. In 2003 he started to work in collaboration with neuroscientists on the problem of multisensory and sensorimotor integration. From 2006 to 2007 he joined the Brain and Research Center (CerCo) of Toulouse, as a temporary researcher. Since 2011 he is leading the Gepetto group of LAASCNRS, specialist of the movement of anthropomorphic systems. His research interests include optimal control, nonlinear control, sensor-based control, human-motion modeling, neurosciences and biomechanics.

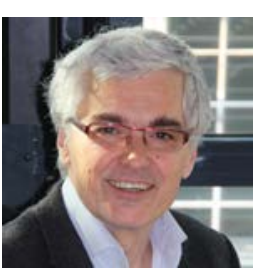

Jean-Paul Laumond IEEE Fellow, is a roboticist. $\mathrm{He}$ is Directeur de Recherche at LAAS-CNRS (team Gepetto) in Toulouse, France. His research is devoted to robot motion. In the early 2000's he created and managed Kineo CAM, a spin-off company from LAAS-CNRS devoted to develop and market motion planning technology. Siemens acquired Kineo CAM in 2012. In 2006, he launched the research team Gepetto dedicated to Human Motion studies along three perspectives: artificial motion for humanoid robots, virtual motion for digital actors and mannequins, and natural motions of human beings. He teaches Robotics at Ecole Normale Suprieure in Paris. He has edited four books. He has published more than 150 papers in international journals and conferences in Robotics, Computer Science, Automatic Control and more recently in Neurosciences. He has been the 2011-2012 recipient of the Chaire Innovation technologique Liliane Bettencourt at Collge de France in Paris. His current project Actanthrope (ERC-ADG 340050) is devoted to the computational foundations of anthropomorphic action.

\footnotetext{
${ }^{2}$ Given a point $Q=\left(\rho_{Q}, \psi_{Q}\right)$ on a logarithmic spiral rotating around point $O_{W}$, the characteristic angle is the angle between the tangent to the spiral in $Q$ and the radial line at the point $Q$.
} 